\title{
BMJ Open Discovery of biomarkers for the presence and progression of left ventricular diastolic dysfunction and HEart faiLure with Preserved ejection Fraction in patients at risk for cardiovascular disease: rationale and design of the HELPFul case-cohort study in a Dutch cardiology outpatient clinic
}

To cite: Valstar GB, Bots SH, Groepenhoff $\mathrm{F}$, et al. Discovery of biomarkers for the presence and progression of left ventricular diastolic dysfunction and HEart faiLure with Preserved ejection Fraction in patients at risk for cardiovascular disease: rationale and design of the HELPFul case-cohort study in a Dutch cardiology outpatient clinic. BMJ Open 2019;9:e028408. doi:10.1136/ bmjopen-2018-028408

- Prepublication history and additional material for this paper are available online. To view these files, please visit the journal online (http://dx.doi. org/10.1136/bmjopen-2018028408).

Received 6 December 2018 Revised 24 April 2019 Accepted 25 April 2019

\section{Check for updates}

(C) Author(s) (or their employer(s)) 2019. Re-use permitted under CC BY-NC. No commercial re-use. See rights and permissions. Published by BMJ.

For numbered affiliations see end of article.

\section{Correspondence to} Dr Hester M den Ruijter; h.m.denRuijter-2@umcutrecht. $\mathrm{nl}$

Gideon B Valstar, ${ }^{1}$ Sophie H Bots, ${ }^{1}$ Floor Groepenhoff, ${ }^{2}$ Aisha Gohar, ${ }^{1}$ Frans H Rutten, ${ }^{3}$ Tim Leiner, ${ }^{4}$ Maarten Jan Maria Cramer, ${ }^{5}$ Arco J Teske, ${ }^{5}$ Leonardo P Suciadi, ${ }^{6}$ Roxana Menken, ${ }^{7}$ Gerard Pasterkamp, ${ }^{2}$ Folkert W Asselbergs, ${ }^{5,8}$ Leonard Hofstra, ${ }^{7,9}$ Michael L Bots, ${ }^{3}$ Hester M den Ruijter ${ }^{1}$

\section{ABSTRACT}

Introduction Left ventricular diastolic dysfunction (LVDD) is a common condition in both sexes that may deteriorate into heart failure (HF) with preserved ejection fraction $(\mathrm{pEF})$, although this seems to happen more often in women than in men. Both LVDD and HFpEF often go unrecognised, necessitating the discovery of biomarkers that aid both the identification of individuals with LVDD at risk of developing $\mathrm{HF}$ and identification of individuals most likely to benefit from treatment.

Methods and analysis HELPFul is an ongoing casecohort study at a Dutch cardiology outpatient clinic enrolling patients aged 45 years and older without history of cardiovascular disease, who were referred by the general practitioner for cardiac evaluation. We included a random sample of patients and enriched the cohort with cases (defined as an E/e' $\geq 8$ measured with echocardiography). Information about medical history, cardiovascular risk factors, electrocardiography, echocardiography, exercise test performance, common carotid intima-media thickness measurement and standard cardiovascular biomarkers was obtained from the routine care data collected by the cardiology outpatient clinic. Study procedure consists of extensive venous blood collection for biobanking and additional standardised questionnaires. Follow-up will consist of standardised questionnaires by mail and linkage to regional and national registries. We will perform cardiac magnetic resonance imaging and coronary $\mathrm{CT}$ angiography in a subgroup of patients to investigate the extent of macrovascular and microvascular coronary disease.

Ethics and dissemination The study protocol was approved by the Institutional Review Board of the University Medical Center Utrecht. Results will be
Strengths and limitations of this study

- Case-cohort study in novel setting: patients at risk of cardiovascular disease at a Dutch cardiology outpatient clinic positioned between the general practitioner and hospitals.

- Venous blood sampling of multiple types of plasma as well as serum, cells and DNA for biobanking.

Designed to accommodate the changing definitions of left ventricular diastolic dysfunction (LVDD) and (subtypes of) heart failure (HF)

- Initial results show a high prevalence of hypertension, but low prevalence of chronic inflammatory comorbidities, such as diabetes mellitus and overweight, for a study investigating LVDD and HF.

- No information on reason for referral by the general practitioner.

disseminated through national and international conferences and in peer-reviewed journals in cardiovascular disease.

Trial registration NTR6016; Pre-results.

\section{INTRODUCTION}

Cardiac disease is a leading cause of morbidity and mortality in industrialised societies. ${ }^{1}$ There is an urgent need for early detection of structural and functional cardiac abnormalities before coronary events occur or before heart failure (HF) develops. HF has a poor prognosis in both sexes, with data from the 
Netherlands showing high mortality rates 30 days, 1 year and 5 years after first hospital admission for HF (13\%, $32 \%$ and $64 \%$ for men and $14 \%, 33 \%$ and $66 \%$ for women, respectively). Mortality rates increased considerably with age, in both men and women, for example, 1-year mortality was $10.5 \%$ in women aged $25-54$ years and increased to $46.1 \%$ in those aged 85 years and older. ${ }^{2}$ This is alarming because HF is common among elderly in the general population with a median prevalence rate of $11.8 \%{ }^{23} \mathrm{HF}$ can be divided into two types: HF with preserved ejection fraction ( $\mathrm{HFpEF}$ ) with a left ventricular ejection fraction (LVEF) $>50 \%$ and $\mathrm{HF}$ with reduced ejection fraction (HFrEF) with an LVEF $<40 \% .{ }^{4} \mathrm{HF}$ is characterised by insufficient pumping of the heart or close to sufficient at the price of increased left ventricular (LV) filling pressures in rest or during exercise. ${ }^{5}$ Generally HFpEF is characterised by increased LV filling pressures that are caused by concentric remodelling and reduced filling, whereas HFrEF is characterised by a lack of contractility and eccentric remodelling. ${ }^{6} \mathrm{HFpEF}$ is rising in prevalence, but its precursor left ventricular diastolic dysfunction (LVDD) is even more prevalent and can also eventually lead to HFrEF or HF with mid-range ejection fraction (HFmrEF) with an LVEF 40\%-49\%. ${ }^{7}$ Important risk factors for developing LVDD and eventually HFpEF are hypertension, overweight, diabetes mellitus and previous ischaemic heart disease. ${ }^{8}$ Within 4-5years $12 \%-25 \%$ of patients with established LVDD progress to symptomatic HFpEF. ${ }^{9-11}$ However, there is still a knowledge gap as to who will progress from LVDD to HFpEF (or other types of HF), and which drivers are involved in this deterioration. ${ }^{12}$

Interestingly, the prevalence of LVDD is similar in men and women, ${ }^{13} 14$ but women with LVDD seem prone to more often develop HFpEF, while men more often develop HFrEF. ${ }^{15-19}$ This sex difference in progression from LVDD to HFpEF is currently poorly understood and warrants closer attention, especially in the view of early identification of patients at risk for worsening LVDD. ${ }^{201}$ Sex differences in underlying mechanisms may partially explain the observed difference in HFpEF prevalence. Women more often present with coronary microvascular dysfunction (CMD).$^{22}$ CMD may lead to myocardial stiffening and LV filling problems, both features of LVDD and HFpEF. Men more often present with pronounced coronary macrovascular disease, a hallmark of HFrEF. ${ }^{22}$

To expand the current knowledge about LVDD and HFpEF, it is important to better understand this underlying heterogeneity. ${ }^{23}$ One promising method to differentiate heterogeneous groups within HFpEF is by detailed 'mapping' of the different phenotypes using both clinical information and biomarkers. Ideally, these biomarkers reflect different pathophysiological processes at the tissue level. Another method may be the application of 'omics' studies. ${ }^{24}$ Identification of patients prone to progression of LVDD to HFpEF may aid the development of new treatment options for patients with HFpEF. While effective treatment options are available for HFrEF and some show
Box 1 Inclusion and exclusion criteria for the HELPFul study

Inclusion: a patient must meet criteria 1, 2 and either 3 or 4Inclusion: a patient must meet criteria 1, 2 and either 3 or 4

1. Age $\geq 45$ years.

2. Written informed consent.

3. E/e' $\geq 8.0$ (selectively for those included as a case).

4. Consultation on a random sample day.

Exclusion: a patient cannot be included in case of any of the criteria belowExclusion: a patient cannot be included in case of any of the criteria below

1. Any past cardiac intervention.

2. Congenital heart disease.

effectiveness in patients with $\mathrm{HFmrEF},{ }^{25}{ }^{26}$ these treatments are ineffective for patients with $\mathrm{HFpEF},{ }^{27}$ although spironolactone might yet prove to be beneficial. ${ }^{28-30}$ At the moment, only aggressive preventive treatment focused on managing hypertension, overweight, diabetes mellitus and a more active lifestyle seem to be effective in reverting or slowing the progression of LVDD to HFpEF. ${ }^{7}$

The aim of the HELPFul study is to discover sex-specific biomarkers for LVDD and HFpEF using several approaches such as multimarker panels and 'omics' studies in combination with extensive additional coronary phenotyping with cardiac magnetic resonance (CMR) imaging and coronary CT angiography (CCTA) in high-risk individuals.

\section{METHODS AND ANALYSIS \\ Study design}

HELPFul is a single-centre, prospective, case-cohort study conducted at a cardiology outpatient clinic in Utrecht, the Netherlands. All patients aged 45 years and older, without previous cardiac interventions or congenital heart disease, who are referred by the general practitioner (GP) to this outpatient clinic are eligible for inclusion. On three of the four inclusion days, only patients with elevated LV filling pressures, defined as an E/e' $\geq 8.0$ are eligible for inclusion. On the fourth day, $25 \%$ of all patients attending that day are invited to participate regardless of their echocardiography results (box 1). The case-cohort design results in a group of 'cases' that have slightly elevated LV filling pressures, of whom a percentage may eventually deteriorate in diastolic function. Part of the patients in the case group may also already have LVDD. The random sample will reflect the distribution of exposure and also serve as a pool for the selection of healthy controls. With a case-cohort design the distribution of LVDD and HFpEF in the source population is accurately reflected in the random sample, while simultaneously creating a pool for the selection of controls. ${ }^{31}$ A flow chart of the study design and procedures is presented in online supplemental figure S1. 


\section{Study population}

Participants are enrolled at the Cardiology Center Utrecht (CCU), one of the outpatient clinics of the Cardiology Center Netherlands. Recruitment of participants started 19 September 2016 and will continue until July 2019. CCU covers the area of the city of Utrecht and neighbouring cities and towns, and on average receives 10-20 newly referred patients per day. The study population comprises adults in the Netherlands aged 45 years and older referred for cardiac evaluation by their GP. Only patients without previous cardiac surgery, a previous cardiac intervention or congenital heart disease are eligible for inclusion. The following cardiac surgical procedures or interventions are considered exclusion criteria: angioplasty, bypass surgery, heart valve surgery or intervention, implantable cardiac defibrillator and/or cardiac resynchronisation therapy, radiofrequency ablation, LV assist device, heart transplantation and pacemaker. Patients referred for preoperative screening or by other specialists than the GP, such as insurance physicians or company physicians, are also excluded from participation.

\section{Rationale for study setting}

The CCU cardiology outpatient clinic is positioned between the GP and the hospital and is intended for quick referral and fast diagnostics to serve the GP, which results in a population at this centre with fewer symptoms and lower cardiovascular disease risk than the population often seen in a similar setting at the hospital. Within this population, we expect large variety in diastolic function, ranging from normal diastolic function to definite LVDD and HFpEF. HFpEF is a syndrome that often presents with intermittent complaints of dyspnoea and/or other less typical symptoms, which can be difficult to detect, especially in elderly patients in the community. GPs are usually the first clinicians who see these patients with unexplained symptoms. Due to the efficient workflow of the cardiac outpatient clinic it is possible to perform this diagnostic workup for 10-20 new patients each day.
There is no waiting list and patients can often be seen within days, even if their complaints are not urgent. This makes referral to this cardiac outpatient clinic a fast and convenient option for the GP. Due to this unique setting the source population of the HELPFul study is different from patients who are referred to the hospital. It therefore provides a unique opportunity to study risk factors and biomarker levels in patients who have not developed LVDD or HFpEF yet, or are still in the early stages of LVDD or HFpEF.

\section{Study end points}

The primary end point is a diagnosis of LVDD or HFpEF. The primary end point is adjudicated by a panel of three experts based on all available diagnostic information and the current recommendations from the 2016 European Society of Cardiology (ESC) guidelines for the diagnosis and treatment of acute and chronic heart failure. ${ }^{532-35}$ The expert panel will comprise two of four available cardiologists (MJMC, AT, RM, LPS) and a GP specialised in HF (FR). Adjudicating the end point by an expert panel is considered the preferred method as a sufficiently reliable reference standard for HFpEF is lacking. ${ }^{36}$ If the diagnostic criteria for LVDD or HFpEF change over time, we will incorporate these new criteria in the classification of LVDD or HFpEF in the HELPFul study population.

Secondary end points are (i) hospitalisation for HF, (ii) a composite end point of cardiovascular death (due to myocardial infarction, stroke, HF, peripheral arterial disease and sudden death of unspecified cause) and (iii) all-cause mortality.

\section{Definition of LVDD/HFpEF}

HFpEF is initially defined according to recommendations of the 2016 ESC guidelines for the diagnosis and treatment of acute and chronic heart failure (table 1). Participants with (i) symptoms or signs typical of $\mathrm{HF}$ and/or brain natriuretic peptide (BNP) $>35 \mathrm{pg} / \mathrm{mL}$, (ii) in combination with an LVEF $>50 \%$ and (iii) objective echocardiographic

\begin{tabular}{|c|c|c|c|}
\hline Type of HF & HFrEF & HFmREF & HFpEF \\
\hline \multicolumn{4}{|l|}{ Criteria } \\
\hline 1 & Symptoms and/or signs $†$ & Symptoms and/or signs $†$ & Symptoms and/or signs $\dagger$ \\
\hline 2 & LVEF $<40 \%$ & LVEF $40 \%-49 \%$ & LVEF $\geq 50 \%$ \\
\hline 3 & - & $\begin{array}{l}\text { 1. Elevated levels of BNP } \neq \\
\text { 2. At least one additional criterion: } \\
\text { a. Relevant structural heart disease } \\
\text { (LVH and/or LA enlargement), } \\
\text { b. Diastolic dysfunction }\end{array}$ & $\begin{array}{l}\text { 1. Elevated levels of BNP } \neq \\
\text { 2. At least one additional criterion: } \\
\text { a. Relevant structural heart disease } \\
\text { (LVH and/or LA enlargement), } \\
\text { b. Diastolic dysfunction }\end{array}$ \\
\hline
\end{tabular}

\footnotetext{
${ }^{*}$ Adapted from 2016 ESC guidelines for acute and chronic heart failure. ${ }^{5}$

†Signs may not be present in early stage of HF (especially in HFpEF) and in patients treated with diuretics. $\ddagger \mathrm{BNP}>35 \mathrm{pg} / \mathrm{mL}$.

BNP, B-type natriuretic peptide; ESC, European Society of Cardiology; HF, heartfailure; HFrEF, heart failure with reduced ejection fraction; HFmREF, heartfailure with mid-range ejection fraction; HFpEF, heartfailure with preserved ejection fraction; LA, left atrial; LVH, left ventricular hypertrophy.
} 
evidence of LVDD will be classified as having HFpEF. ${ }^{5}$ The presence of these criteria but with an LVEF between $40 \%$ and $49 \%$ or an LVEF $<40 \%$ will result in participants being classified as having HFmrEF and HFrEF, respectively (table 1). LVDD is defined as $\mathrm{E} / \mathrm{e}^{\prime}$ ratio $>13$ or $\mathrm{E} / \mathrm{e}^{\prime}$ between 8 and 13 with other structural abnormalities, such as left atrial volume index (LAVi) $>34 \mathrm{~mL} / \mathrm{m}^{2}$, or left ventricular mass index $(\mathrm{LVMi})>95 \mathrm{~g} / \mathrm{m}^{2}$ for women and $>115 \mathrm{~g} / \mathrm{m}^{2}$ for men and/or functional abnormalities, such as e' lateral $<10 \mathrm{~m} / \mathrm{s}$ or e' septal $<7 \mathrm{~m} / \mathrm{s}$ or tricuspid regurgitation (TR) velocity $>2.8 \mathrm{~m} / \mathrm{s}^{5}$

\section{Standard care measurements at enrolment}

Standard care measurements consist of history taking, physical examination, ECG (Welch Allyn Cardioperfect Pro recorder), laboratory blood measurements (Roche Reflotron Sprint system), an exercise test on a watt bike (Lode Corival Eccentric) with simultaneous blood pressure measurements (Medtronic BL-6 Compact) and ECG recordings (Welch Allyn Cardioperfect recorder) and transthoracic echocardiography (with a General Electric Vivid E6 or E7 cardiovascular echocardiography device). In addition, comorbidities and current medication use will be registered. Physical examination includes measuring height and weight, blood pressure (two readings at least on a Microlife WatchBP), pulse rate and calculation of the respiratory rate. A standard 12-lead ECG (Welch Allyn Cardioperfect Pro recorder) will be recorded in supine position and interpreted by a cardiologist. The standard laboratory test returns plasma and serum levels of potassium, glucose, haemoglobin, creatinine, total cholesterol, low-density lipoprotein, high-density lipoprotein and triglycerides.

\section{Echocardiography}

Comprehensive transthoracic echocardiographic examinations were performed by trained sonographers and interpreted by a cardiologist in accordance with the European Association of Cardiovascular Imaging 2016 recommendations for chamber quantification. ${ }^{37}$ The LVEF was assessed quantitatively (Teichholz), or semi-quantitatively (eye-balling). Multiple diastolic parameters were measured, including pulsed-wave Doppler of the mitral and pulmonary venous inflow and tissue Doppler imaging of the mitral annulus motion. The ratio of peak early (E) diastolic filling velocity to peak atrial (A) contraction filling velocity was calculated to derive the $\mathrm{E} / \mathrm{A}$ ratio. The early diastolic mitral annular recoil velocity (e') was determined at both the septal and lateral wall. The E/e' ratio was calculated by dividing $\mathrm{E}$ with the average of septal and lateral e'. LAVi was derived from tracing the left atrium during maximal atrial filling in the apical two-chamber and apical four-chamber views and subsequently indexing by body surface area (BSA). LVMi was calculated according to the formula validated by Devereux and subsequently indexed by BSA. ${ }^{38}$ The sonographers assessed TR in the parasternal right ventricular inflow, parasternal shortaxis and apical four-chamber views. A minimum of five sequential complexes were recorded. The peak velocity of the TR signal was measured with continuous-wave Doppler and the systolic pulmonary artery pressure was calculated with the modified Bernoulli's equation. ${ }^{39}$

\section{Cardiologist consult}

The standard care protocol is for the CCU cardiologist to perform an assessment of all information that is collected during diagnostic workup. The cardiologist may wish to extend beyond the standard diagnostic workup procedure when there is a medical indication to do so. Additional information on symptoms may be collected, or the cardiologist may perform additional physical examinations in accordance with the appropriate guidelines. ${ }^{5}$ All data collected during the standard care pathway is recorded in the electronic health database of CCU and will be extracted for HELPFul patients. Aside from the data collected during diagnostic workup, the electronic health database of CCU may contain information on follow-up visits and on results of procedures performed at other locations than CCU.

\section{Specific HELPFul measurements}

Eligible patients are invited to participate in the HELPFul study by their CCU cardiologist at the end of the visit. After obtaining informed consent, a study physician or a trained research nurse takes a detailed questionnaire of the patient at the baseline visit. Data were collected on symptoms (including questions adapted from the Minnesota Living with Heart Failure questionnaire, New York Heart Association classification and Medical Research Council dyspnoea scale), cardiovascular risk factors, general and cardiovascular medical history, family history for cardiovascular events (positive if a first-degree relative had a cardiovascular event at age 65 years or younger), pregnancy history and menopausal status and medication use.

\section{Study-specific blood sampling}

Approximately $70 \mathrm{~mL}$ of venous blood is collected at baseline immediately after obtaining informed consent. After ultracentrifugation, serum, EDTA, citrate and sodium-heparin plasma are aliquoted and frozen at $-80^{\circ} \mathrm{C}$. Peripheral blood mononuclear cells (PBMCs) are isolated from EDTA blood and stored in a CoolCell freezing container to ensure freezing at a standardised controlled rate of $-1{ }^{\circ} \mathrm{C} / \mathrm{min}$ cell in a $-80^{\circ} \mathrm{C}$ freezer. Whole blood EDTA aliquots are frozen at $-80^{\circ} \mathrm{C}$ for the purpose of storing genetic material for genome analyses. All samples are frozen within 2 hours after the blood samples were taken by venipuncture. Both blood and cell samples are transported to the Central BioBank of the University Medical Center Utrecht (UMCU) on a weekly basis for long-term storage of aliquoted samples in $-80^{\circ} \mathrm{C}$ freezers and of PBMC vials in liquid nitrogen tanks. One $2.5 \mathrm{~mL}$ EDTA plasma tube is transported to the clinical chemistry department of the UMCU for haematological analysis by 
an Abbott CELL-DYN Sapphire in order to obtain white blood cell counts and differentials.

\section{Plasma biomarkers}

BNP and high-sensitivity troponin-I (hs-TnI) are measured in plasma of every participant as part of the research protocol. Hs-TnI and BNP are measured using the appropriate assay on the ARCHITECT i2000 analyzer (Abbott Park, Chicago, Illinois, USA).

\section{Assessment for microvascular disease}

As part of a recent (2018) amendment of the HELPFul study, patients presenting at CCU with complaints of chest pain are invited for additional CMR and CCTA. The aim of this project is to improve the phenotyping of the HELPFul population by assessing the prevalence of both (non-) obstructive coronary artery disease (CAD) and CMD. This extensive phenotyping may further aid in identification of subgroups that can be used for biomarker discovery studies.

\section{HELPFul study follow-up}

All participants will be followed up for occurrence of any cardiac event (fatal and non-fatal myocardial infarction, proven unstable angina, coronary revascularisation, hospitalisation for HF, stroke, peripheral arterial disease, sudden death of unspecified cause and death from any cause) by means of:

1. linkage with regional (Julius GPs Network) ${ }^{40}$ and national registries (National Hospital Discharge Registry and Statistics Netherlands (ie, National Causes of Death Registry) $)^{41}$;

2. questionnaires sent through email or letter 2 years after enrolment in HELPFul, after which a yearly questionnaire will be sent. The questionnaires will enquire after status of symptoms of cardiovascular disease and specifically of heart failure and hospitalisation for cardiac disease.

\section{Case-control selection within the case-cohort}

For the case-cohort, we consider cases to be patients with echocardiographic-defined E/e' $\geq 8$. Cohort refers to the total population sample (which could also include patients with $\mathrm{E} / \mathrm{e}^{\prime} \geq 8$ ). In this way, we create a case-cohort design. For a nested diagnostic case-control study, we take samples from this case-cohort study in which the cases are patients with $\mathrm{HFpEF}$, and controls (no HFpEF) are sampled from the cohort. For the power calculations, we use the 'Harrell's rule of thumb' applicable to diagnostic research. For the aforementioned calculation, we speculate that around $15 \%$ of the patients will be diagnosed with HFpEF; the true cases for the nested case-control design. Thus, we can evaluate one diagnostic predictor in multivariable logistic regression analysis per $10 \mathrm{HFpEF}$ cases. As we aim to analyse at least 15 determinants/ biomarkers, we therefore would need at least 150 patients with HFpEF. Hence, we require the inclusion of around 1000 patients in total.

\section{Statistical analyses}

Descriptive data will be presented as frequencies with proportions for categorical variables and either as means with corresponding SD or medians with corresponding IQR for continuous variables depending on the distribution. We will use the appropriate statistical tests for differences at baseline, such as Pearson's $\mathrm{X}^{2}$ test for frequencies, Mann-Whitney $U$ test for non-normally distributed continuous variables and Student's t-test for normally distributed variables. In case of possible interaction of sex, interaction terms will be created for sex and for each variable of interest. The interaction terms will then be tested for significance using logistic regression models.

Logistic regression will be used to analyse the association of various determinants with LVDD and HFpEF status at baseline. Survival analyses will be used to evaluate the relationship between baseline determinants and the primary and secondary study outcomes. All models will be adjusted for suspected confounders. All data analyses will be performed using appropriate statistics software.

\section{Data analysis prediction algorithm}

We will develop a prediction model using state-of-the-art methodology as described in the Transparent Reporting of multivariable prediction model for Individual Prognosis Or Diagnosis (TRIPOD) statement. ${ }^{42}$ Missing data will be imputed to minimise bias and loss of precision. The choice of imputation technique will depend on the extent and type of missing data. The linearity assumption will be tested using restricted cubic splines and transformations will be applied where necessary. A backstep model will be used to eliminate redundant predictors, using the likelihood ratio test with a $\mathrm{p}$ value of 0.157 (equal to Akaike's Information Criterion for predictors with $1 \mathrm{df}$ ). Eliminating redundant predictors reduces the risk of overfitting the prediction model and makes the model easier to use.

The discrimination and calibration of both models will be compared between the overall and final (reduced) prediction model to evaluate their performance. Discrimination is defined as the ability of the model to distinguish between patients who have LVDD and/or HFpEF from those who do not have the outcome and is quantified with the area under the curve (AUC) of a receiver operating characteristic plot. Calibration refers to the agreement between the predicted absolute risks of $\mathrm{HFpEF}$ being present and the observed HFpEF frequencies. This is expressed by comparing the observed incidence of HFpEF per predicted risk category.

Prediction models are known for overestimated regression coefficients, which result in too extreme (optimistic) predictions when applied in new patients. Therefore, we will (internally) validate our model with bootstrapping techniques where in each bootstrap sample the entire modelling process (including the variable selection process) is repeated. The regression coefficients of the final model are then adjusted for overfitting 
by multiplying it with the estimated shrinkage factor, yielded from the bootstrap. The bootstrap procedure is also used to correct the AUC for overfitting, which can be considered as an estimate of discriminative ability that is expected in future patients. These analyses will be performed using the appropriate $\mathrm{R}$ version.

\section{DISCUSSION}

The HELPFul study is currently recruiting men and women at a cardiology outpatient clinic with the double aim of studying LVDD and HFpEF and providing sufficient power for sex-stratified biomarker research. We expect the prevalence of LVDD and HFpEF in the HELPFul study to be similar to the prevalence in other studies that screened patients at the GP practice. ${ }^{3} 344344$ Biomarkers panels derived from discoveries made in the HELPFul study may help to direct screening both at the GP practice and (outpatient) cardiology clinics.

To the best of our knowledge, the evidence on (prediction of) LVDD deterioration is rather limited. While information is available on risk factors for and biomarkers associated with development of HFpEF, ${ }^{9} 1045$ the few studies investigating the development of LVDD did not include biomarkers. ${ }^{9} 1146$ These studies have shown that diastolic function deteriorates in around $25 \%$ of patients with LVDD over the course of 5 years ${ }^{911}$ and that LVDD is an independent risk factor for development of $\mathrm{HF}^{9}{ }^{46}$ Preventing the development of LVDD in this population is therefore highly relevant, because it may prevent many new cases of HF as well. However, current international guidelines on HF do not provide any recommendations on early detection of LVDD and subsequent prevention of HFpEF. As a consequence, current treatment is focused on reduction of symptoms and management of risk factors in patients with LVDD who have already progressed to HFpEF. However, it is generally acknowledged that (drug) interventions could be more effective in the very early phases of HFpEF or LVDD underscoring the relevance of early biomarkers and mechanistic insight. $^{7}$

One of the aims of the HELPFul study is to fill this knowledge gap by focusing on biomarkers that may aid the prediction of LVDD deterioration. We will use follow-up echocardiography measurements to investigate drivers associated with progression of LVDD and development of HFpEF in the participants of the HELPFul study. In addition, linkage to nationwide hospitalisation registers and death registers will enable the assessment of the clinical consequences of LVDD and HFpEF.

To investigate the possible connection between microvascular disease and LVDD and HFpEF, the prevalence of (non-)obstructive $\mathrm{CAD}$ and $\mathrm{CMD}$ will be assessed using CMR and CCTA imaging. A meta-analysis of international variations in angina prevalence across 31 countries showed a slightly higher prevalence of angina in women with a pooled sex ratio of $1.20 .{ }^{47}$ Other studies have suggested that this could be driven by CMD instead of obstructive CAD of the epicardial vessels. ${ }^{48}{ }^{49}$ Furthermore, in a cohort of women with chest pain suspected for obstructive CAD, those who were hospitalised for HF at 6-year follow-up suffered predominantly from HFpEF. ${ }^{50}$

The HELPFul study is designed to accommodate changes in the definitions of LVDD and HFpEF, HFrEF and HFmrEF, which have occurred frequently over the past 10 years. At the moment, multiple guidelines for $\mathrm{HF}^{51}$ provide different criteria and cut-offs for LVDD and HFpEF. ${ }^{52} 53$ This has resulted in varying recommendations over the last decades, which hampers the comparison of diagnostic studies of LVDD and HFpEF. ${ }^{16}$

\section{Limitations}

1. The cases as defined in the study design are not necessarily patients with LVDD or HFpEF by current diagnostic criteria, but were selected for having slightly elevated LV filling pressures on echocardiography. As explained in the discussion, there is a knowledge gap on which patients with a possible early form of LVDD will eventually progress towards actual LVDD. Therefore, we consider sampling these patients to investigate biomarker levels and risk factors involved in progression to LVDD of high value. However, we are sampling relatively many participants with no definitive diagnosis of LVDD or HFpEF by current diagnostic criteria. However, this will predominantly lead to a lower efficiency for selection of patients with LVDD or $\mathrm{HFpEF}$, whereas it does provide the opportunity to efficiently study patients most at risk of eventually developing LVDD or HFpEF.

2. Initial analyses indicate that the HELPFul study population has a high prevalence of hypertension, but a low prevalence of chronic inflammatory comorbidities that are associated with LVDD and HFpEF, such as diabetes mellitus and overweight. Furthermore, chest pain is a common complaint, hinting towards the importance of possible CMD in this study population. The external validity could be affected when the generalisability to the population at risk of developing LVDD or HF$\mathrm{pEF}$ in the community is lower. However, hypertension and CMD are known to be important in the development of LVDD and HFpEF. Therefore, we consider the HELPFul study population to be representative of, for instance, community-based elderly individuals of 65 years and older. Even so prudence is appropriate when generalising results to community-based or hospital-based patients with LVDD or HFpEF, particularly in the context of chronic inflammatory comorbidities.

3. Patients are referred to this centre when the GP considers referral to be indicated. However, the cardiology outpatient clinic does not record the indication for referral in their electronic patient database. Therefore, we have no data on the indication for referral. As mentioned previously, patients who are referred often do not have acute/severe complaints of cardiac problems. This could lead to referral bias, which is a form of selection bias usually affecting the comparison of cases 
and controls and generalisability of the results. Due to our study design, we do not expect referral bias to be a problem, because cases and participants of the random sample are selected from the same source population. Furthermore, if future results are generalised within the context of the study, we do not see a problem with external validity.

In conclusion, HELPFul is an ongoing study recruiting men and women at a cardiology outpatient clinic with the aim of studying LVDD and HFpEF, that offers unique opportunities for well-powered biomarker research in a sex-stratified manner.

\section{Ethics and dissemination}

The study is conducted according to the principles of the Declaration of Helsinki October 2013 and in accordance with the Medical Research Involving Human Subjects Act. The study does not interfere with routine patient care, and all patients will be treated at the discretion of their cardiologist or GP according to the appropriate guidelines. Participants have given informed consent for extra venous blood collection and storage, and for additional questionnaires. We will present our findings at national and international conferences and in peer-reviewed journals in cardiovascular disease.

\section{Data safety and monitoring board}

An independent data and safety monitoring board was not installed.

\section{Author affiliations}

${ }^{1}$ Laboratory of Experimental Cardiology, University Medical Center Utrecht, Utrecht University, Utrecht, The Netherlands

${ }^{2}$ Department of Clinical Chemistry and Hematology, University Medical Center Utrecht, Utrecht University, Utrecht, The Netherlands

${ }^{3}$ Julius Center for Health Sciences and Primary Care, University Medical Center Utrecht, Utrecht University, Utrecht, The Netherlands

${ }^{4}$ Department of Radiology, University Medical Centre Utrecht, Utrecht University, Utrecht, The Netherlands

${ }^{5}$ Department of Cardiology, University Medical Center Utrecht, Utrecht University, Utrecht, The Netherlands

${ }^{6}$ Department of Cardiology, Siloam Heart Institute, Siloam Hospital KebonJeruk, Jakarta, Indonesia

${ }^{7}$ Cardiologie Centra Nederland, Utrecht, The Netherlands

${ }^{8}$ Institute of Cardiovascular Science and Institute of Health Informatics, Faculty of Population Health Sciences, University College London, London, United Kingdom ${ }^{9}$ Department of Cardiology, Vu University Medical Center, Amsterdam, The Netherlands

Acknowledgements The authors would like to thank Jonne Hos for her outstanding efforts in and support of the HELPFul study. The authors would like to thank Evelyn Velema, Merel Schurink, Anouk Eikendal and Marja Maat-Leersum for their contribution to the recruitment of patients and their aid in validating and cleaning the raw data. The authors would like to thank Arjan Schoneveld, Danny Elbersen, Sander van der Weg, Daniek Kapteijn, Nanique Tulkens, Naomi Parr, Hemze Al-Khamisi and Lianne Granneman for their contribution to workup of the samples stored in the biobank. The authors would also like to thank all people involved from Cardiology Center Netherlands and specifically the team at Cardiology Center Utrecht for their assistance.

Contributors GBV: acquisition of data, analysis and interpretation of data, drafting and revising the manuscript. SHB: acquisition of data, revising the manuscript. FG: addition of imaging to HELPFul study, acquisition of data, revising the manuscript. AG: acquisition of data, revising the manuscript. FHR: conception and study design, interpretation of data, participation in expert panel, drafting and revising the manuscript. TL: addition of imaging to HELPFul study, revising the manuscript. MJMC: interpretation of data, participation in expert panel, revising the manuscript. AJT: participation in expert panel, revising the manuscript. LPS: participation in expert panel, quality control of raw echocardiography data, revising the manuscript. RM: addition of imaging to HELPFul study, participation in expert panel, revising the manuscript. GP: conception and study design, revising the manuscript. FWA: conception and study design, interpretation of data, revising the manuscript. LH: conception and study design, revising the manuscript. MLB: conception and study design, analysis and interpretation of data, drafting and revising the manuscript. HMdR: conception and study design, analysis and interpretation of data, addition of imaging to HELPFul study, drafting and revising the manuscript. All authors have read and given final approval of the submitted manuscript.

Funding This investigator-initiated project received funding from the Dutch Heart Foundation 2013T084 (Queen of Hearts) and the first author (GV) is supported by Dutch Heart Foundation Grant CVON2014-11 RECONNECT. Additional phenotyping using CMR and CCTA is supported by funding from CVON 2017-22 ARGUS. Linkage to regional and national registries is supported by funding from Netherlands Organization for Health Research and Development (ZonMW) PRIDE (849100003). FA is supported by UCL Hospitals NIHR Biomedical Research Centre.

Competing interests None declared.

Patient consent for publication Not required.

Ethics approval The study protocol is approved by the Institutional Review Board of the University Medical Center Utrecht.

Provenance and peer review Not commissioned; externally peer reviewed.

Open access This is an open access article distributed in accordance with the Creative Commons Attribution Non Commercial (CC BY-NC 4.0) license, which permits others to distribute, remix, adapt, build upon this work non-commercially, and license their derivative works on different terms, provided the original work is properly cited, appropriate credit is given, any changes made indicated, and the use is non-commercial. See: http://creativecommons.org/licenses/by-nc/4.0/.

\section{REFERENCES}

1. Mozaffarian D, Benjamin EJ, Go AS, et al. Executive summary: heart disease and stroke statistics--2016 update: a report from the American Heart Association. Circulation 2016;133:447-54.

2. Buddeke J, Valstar GB, Dis van I, et al. Prognose na ziekenhuisopname voor hartfalen. Hart- en vaatziekten in Nederland 2017, cijfers over leefstijl, risicofactoren, ziekte en sterfte. Den Haag: Hartstichting, 2017:61-70.

3. van Riet EE, Hoes AW, Wagenaar KP, et al. Epidemiology of heart failure: the prevalence of heart failure and ventricular dysfunction in older adults over time. A systematic review. Eur J Heart Fail 2016;18:242-52.

4. Owan TE, Hodge DO, Herges RM, et al. Trends in prevalence and outcome of heart failure with preserved ejection fraction. $N$ Engl $J$ Med 2006;355:251-9.

5. Ponikowski P, Voors AA, Anker SD, et al. ESC Guidelines for the diagnosis and treatment of acute and chronic heart failure. Eur Heart J 2016;2016:2129-200.

6. Rutten FH, Clark AL, Hoes AW. How big a problem is heart failure with a normal ejection fraction? BMJ 2016;353:i1706.

7. Wan $\mathrm{SH}$, Vogel MW, Chen $\mathrm{HH}$. Pre-clinical diastolic dysfunction. $J$ Am Coll Cardiol 2014;63:407-16.

8. Paulus WJ, Tschöpe C. A novel paradigm for heart failure with preserved ejection fraction: comorbidities drive myocardial dysfunction and remodeling through coronary microvascular endothelial inflammation. J Am Coll Cardiol 2013;62:263-71.

9. Kane GC, Karon BL, Mahoney DW, et al. Progression of left ventricular diastolic dysfunction and risk of heart failure. JAMA 2011;306:856-63.

10. Vogel MW, Slusser JP, Hodge DO, et al. The natural history of preclinical diastolic dysfunction: a population-based study. Circ Heart Fail 2012;5:144-51.

11. Achong N, Wahi S, Marwick TH. Evolution and outcome of diastolic dysfunction. Heart 2009;95:813-8.

12. Komajda M, Lam CS, Csp L. Heart failure with preserved ejection fraction: a clinical dilemma. Eur Heart J 2014;35:1022-32.

13. Redfield MM, Jacobsen SJ, Burnett, Jr JC, et al. Burden of systolic and diastolic ventricular dysfunction in the community. JAMA 2003;289:194.

14. Mureddu GF, Agabiti N, Rizzello V, et al. Prevalence of preclinical and clinical heart failure in the elderly. A population-based study in Central Italy. Eur J Heart Fail 2012;14:718-29. 
15. Dunlay SM, Roger VL, Redfield MM. Epidemiology of heart failure with preserved ejection fraction. Nat Rev Cardiol 2017;14:591-602.

16. Bouthoorn S, Valstar GB, Gohar A, et al. The prevalence of left ventricular diastolic dysfunction and heart failure with preserved ejection fraction in men and women with type 2 diabetes: a systematic review and meta-analysis. Diab Vasc Dis Res 2018;15:477-93.

17. Brouwers FP, de Boer RA, van der Harst $P$, et al. Incidence and epidemiology of new onset heart failure with preserved vs. reduced ejection fraction in a community-based cohort: 11-year follow-up of PREVEND. Eur Heart J 2013;34:1424-31.

18. Lee DS, Gona P, Vasan RS, et al. Relation of disease pathogenesis and risk factors to heart failure with preserved or reduced ejection fraction: insights from the framingham heart study of the national heart, lung, and blood institute. Circulation 2009;119:3070-7.

19. Bouthoorn S, Gohar A, Valstar G, et al. Prevalence of left ventricular systolic dysfunction and heart failure with reduced ejection fraction in men and women with type 2 diabetes mellitus: a systematic review and meta-analysis. Cardiovasc Diabetol 2018;17:58.

20. Scantlebury DC, Borlaug BA, a BB. Why are women more likely than men to develop heart failure with preserved ejection fraction? Curr Opin Cardiol 2011;26:562-8.

21. Eikendal ALM, Gohar A, Rutten FH, et al. Sex-Specific relations of cardiovascular risk factors with left ventricular diastolic dysfunction/ heart failure with preserved ejection fraction are underreported: a call for action. J Card Fail 2018:24:412-4.

22. Sharma K, Al Rifai M, Ahmed HM, et al. Usefulness of coronary artery calcium to predict heart failure with preserved ejection fraction in men versus women (from the Multi-Ethnic Study of Atherosclerosis). Am J Cardiol 2017:120:1847-53.

23. Shah SJ, Katz DH, Selvaraj S, et al. Phenomapping for novel classification of heart failure with preserved ejection fraction. Circulation 2015;131:269-79.

24. Toma M, Mak GJ, Chen V, et al. Differentiating heart failure phenotypes using sex-specific transcriptomic and proteomic biomarker panels. ESC Heart Fail 2017;4:301-11.

25. Cleland JGF, Bunting KV, Flather MD, et al. Beta-blockers for heart failure with reduced, mid-range, and preserved ejection fraction: an individual patient-level analysis of double-blind randomized trials. Eur Heart $J$ 2018;39:26-35.

26. Lund LH, Claggett B, Liu J, et al. Heart failure with mid-range ejection fraction in CHARM: characteristics, outcomes and effect of candesartan across the entire ejection fraction spectrum. Eur $\mathrm{J}$ Heart Fail 2018:20:1230-9.

27. Holland DJ, Kumbhani DJ, Ahmed SH, et al. Effects of treatment on exercise tolerance, cardiac function, and mortality in heart failure with preserved ejection fraction. A meta-analysis. J Am Coll Cardiol 2011;57:1676-86.

28. Pitt B, Pfeffer MA, Assmann SF, et al. Spironolactone for heart failure with preserved ejection fraction. N Engl J Med 2014;370:1383-92.

29. Mitter SS, Shah SJ. Spironolactone for management of heart failure with preserved ejection fraction: whither to after TOPCAT? Curr Atheroscler Rep 2015;17:64.

30. Anand IS, Claggett B, Liu J, et al. Interaction between spironolactone and natriuretic peptides in patients with Heart Failure and Preserved Ejection Fraction: From the TOPCAT Trial. JACC Heart Fail 2017;5:241-52

31. Prentice RL. A case-cohort design for epidemiologic cohort studies and disease prevention trials. Biometrika 1986;73:1-11.

32. Reddy YNV, Carter RE, Obokata M, et al. A Simple, Evidence-Based Approach to Help Guide Diagnosis of Heart Failure With Preserved Ejection Fraction. Circulation 2018;138:861-70.

33. Cowie MR, Wood DA, Coats AJ, et al. Incidence and aetiology of heart failure; a population-based study. Eur Heart $J$ 1999;20:421-8.

34. Rutten FH, Moons KG, Cramer MJ, et al. Recognising heart failure in elderly patients with stable chronic obstructive pulmonary disease in primary care: cross sectional diagnostic study. BMJ 2005;331:1379.

35. Bossuyt PM, Reitsma JB, Bruns DE, et al. Towards complete and accurate reporting of studies of diagnostic accuracy: the STARD initiative. BMJ 2003;326:41-4.
36. Kelder JC, Cramer MJ, van Wijngaarden J, et al. The diagnostic value of physical examination and additional testing in primary care patients with suspected heart failure. Circulation 2011;124:2865-73.

37. Lang RM, Badano LP, Mor-Avi V, et al. Recommendations for cardiac chamber quantification by echocardiography in adults: an update from the American Society of Echocardiography and the European Association of Cardiovascular Imaging. Eur Heart J Cardiovasc Imaging 2015;16:233-71.

38. Devereux RB, Alonso DR, Lutas EM, et al. Echocardiographic assessment of left ventricular hypertrophy: comparison to necropsy findings. Am J Cardiol 1986;57:450-8.

39. Rutten $\mathrm{FH}$, Cramer MJ, Grobbee DE, et al. Unrecognized heart failure in elderly patients with stable chronic obstructive pulmonary disease. Eur Heart J 2005;26:1887-94.

40. Sollie A, Roskam J, Sijmons RH, et al. Do GPs know their patients with cancer? Assessing the quality of cancer registration in Dutch primary care: a cross-sectional validation study. BMJ Open 2016;6:1-10

41. Buddeke J, Bots ML, van Dis I, et al. Trends in comorbidity in patients hospitalised for cardiovascular disease. Int $J$ Cardiol 2017;248:382-8.

42. Moons KG, Altman DG, Reitsma JB, et al. Transparent reporting of a multivariable prediction model for Individual Prognosis or Diagnosis (TRIPOD): explanation and elaboration. Ann Intern Med 2015;162:55-65.

43. van Riet EE, Hoes AW, Limburg A, et al. Prevalence of unrecognized heart failure in older persons with shortness of breath on exertion. Eur J Heart Fail 2014;16:772-7.

44. Boonman-de Winter LJ, Rutten FH, Cramer MJ, et al. High prevalence of previously unknown heart failure and left ventricular dysfunction in patients with type 2 diabetes. Diabetologia 2012;55:2154-62.

45. Correa de Sa DD, Hodge DO, Slusser JP, et al. Progression of preclinical diastolic dysfunction to the development of symptoms. Heart 2010;96:528-32.

46. Echouffo-Tcheugui JB, Erqou S, Butler J, et al. Assessing the risk of progression from asymptomatic left ventricular dysfunction to overt heart failure: a systematic overview and meta-analysis. JACC Heart Fail 2016;4:237-48.

47. Hemingway $\mathrm{H}$, Langenberg $\mathrm{C}$, Damant $\mathrm{J}$, et al. Prevalence of angina in women versus men: a systematic review and metaanalysis of international variations across 31 countries. Circulation 2008;117:1526-36.

48. Bairey Merz CN, Shaw LJ, Reis SE, et al. Insights from the NHLBISponsored Women's Ischemia Syndrome Evaluation (WISE) Study: Part II: gender differences in presentation, diagnosis, and outcome with regard to gender-based pathophysiology of atherosclerosis and macrovascular and microvascular coronary disease. J Am Coll Cardiol 2006;47:S21-9.

49. Shaw LJ, Bairey Merz CN, Pepine CJ, et al. Insights from the NHLBI-Sponsored Women's Ischemia Syndrome Evaluation (WISE) study: part i: gender differences in traditional and novel risk factors, symptom evaluation, and gender-optimized diagnostic strategies. $J$ Am Coll Cardiol 2006;47:S4-20.

50. Bakir M, Nelson MD, Jones E, et al. Heart failure hospitalization in women with signs and symptoms of ischemia: a report from the women's ischemia syndrome evaluation study. Int $J$ Cardiol 2016;223:936-9.

51. Nagueh SF, Smiseth OA, Appleton CP, et al. Recommendations for the evaluation of left ventricular diastolic function by echocardiography: an update from the American Society of Echocardiography and the European Association of Cardiovascular Imaging. J Am Soc Echocardiogr 2016;29:277-314.

52. Nagueh SF, Appleton CP, Gillebert TC, et al. Recommendations for the evaluation of left ventricular diastolic function by echocardiography. J Am Soc Echocardiogr 2009;22:107-33.

53. McMurray JJ, Adamopoulos S, Anker SD, et al. ESC guidelines for the diagnosis and treatment of acute and chronic heart failure 2012: The Task Force for the Diagnosis and Treatment of Acute and Chronic Heart Failure 2012 of the European Society of Cardiology. Developed in collaboration with the Heart Failure Association (HFA) of the ESC. Eur J Heart Fail 2012;14:803-69. 